- Electronic data logging verified the level of compliance with toothbrushing regimens.

- Large differences were observed between recorded (electronic loggers) and reported (diaries) levels of compliance.

- Clinicians should always take account of issues of compliance relating to the success or failure of any clinical intervention or therapy.

\title{
Assessing adherence with toothbrushing instructions using a data logger toothbrush
}

\author{
G. McCracken, ${ }^{1}$ J. Janssen, ${ }_{1}^{2}$ L. Heasman, ${ }_{1}^{3}$ F. Stacey ${ }_{1}{ }^{4}$ N. Steen, ${ }_{1}{ }^{5}$ M. deJager ${ }^{6}$ and P. Heasman ${ }^{7}$
}

\begin{abstract}
Objectives To evaluate patient compliance with toothbrushing instructions using a data logger and a brushing diary.

Methods Dental patients were provided with powered toothbrushes modified to carry electronic data loggers. Demonstration of the most effective way to use the toothbrush was provided with instructions to brush for 2 minutes morning and evening. Data logger brushes were supplied for two episodes of 8 weeks. Brushing time was also recorded in a diary during episode 1.

Results Data from 14 brushes (2,287 recordings) were used to evaluate compliance in episode 1. Nine brushes (1,526 recordings) were used in episode 2. A total of 2,333 brushing events were reported manually in the diaries. Data logger records during episode 1 showed that only $34 \%$ of events were compliant with the instruction of a 2 minute brushing time, $20 \%$ were partially compliant (within \pm 30 s of 2 minutes), and $46 \%$ of events were non-compliant (>30s from 2 minutes). The respective proportions for episode 2 were 24\%, 24\% and 52\%. Diary data reported $58 \%$ of events as compliant with $42 \%$ non-compliant.

Conclusions Manually completed brushing diaries do not provide an accurate reflection of subjects' compliance with toothbrushing instructions.
\end{abstract}

\section{INTRODUCTION}

The impact of subject behaviour upon the outcome of any therapy can be profound, although difficult to quantify. Issues relating to compliance with professional advice and therapies have been discussed widely, ${ }^{1-3}$ with, for example: diaries, pill counts, or biological markers used to measure this variable. In a review, Urquhart ${ }^{4}$ suggested that the true level of compliance was probably a great deal poorer than the above methods would reveal. Wilson suggested that compliance tended to be poor in subjects who have chronic diseases

\footnotetext{
1*Lecturer and Honorary Specialist Registrar, ${ }^{3,4}$ Research Dental Hygienist, ${ }^{7}$ Professor of Periodontology and Honorary Consultant, School of Dental Sciences, University of Newcastle upon Tyne, ${ }^{2}$ Research Engineer, Philips Research, Personal Care Institute, Eindhoven, The Netherlands, ${ }^{5}$ Principal Research Associate, Centre for Health Services Research, University of Newcastle upon Tyne, ${ }^{6}$ Director Innovation and Methodology, Clinical and Scientific Affairs, Philips Oral Healthcare, Snoqualmie, WA, USA, *Correspondence to: Dr Giles McCracken, School of Dental Sciences, University of Newcastle upon Tyne, Framlington Place, Newcastle upon Tyne NE2 4BW Email: g.i.mccracken@ncl.ac.uk
}

that are perceived as non-threatening, and, even in the most optimistic studies, the rate of compliance with suggested toothbrushing was less than 50\%. Even when non-compliance may have fatal consequences, adhering to professional guidance cannot be assured. An accepted generalisation suggests that only one-third of subjects comply adequately with a chosen therapy, with one-third who do not comply at all. ${ }^{6}$

If less than half of a study population are likely to comply with professional advice, it is essential to determine any effect that noncompliant behaviour may have upon the interpretation of clinical trial data. Evaluation of subject compliance with professional instruction can be difficult. For example, a dental subject who makes a 'special' effort with oral hygiene practices on the day of a clinical examination can easily mask poor compliance, particularly if dental plaque levels are used as the outcome measure. This type of behaviour has been described as 'white-coat' compliance. ${ }^{7-10} \mathrm{~A}$ number of oral health studies have attempted to assess levels of compliance by asking subjects to complete brushing diaries or calendars ${ }^{11-15}$ or by using questionnaires. ${ }^{6-20}$ However, these methods of assessment only allow reported activity rather than actual recorded activity.

Since the 1970s an alternative to direct supervision and/or reported compliance has been available. Electronic data loggers have been used with great effect to record patterns of behaviour within pharmaceutical clinical trials. ${ }^{21-23}$ These devices have developed significantly into highly sophisticated tools to monitor behaviour and compliance. With contemporary electronics and power sources it is now possible to integrate data loggers easily within medical devices including, for example powered toothbrushes (PTBs).

The aims of this study were:

- to estimate the compliance of a group of dental subjects with brushing time instructions for the daily use of a PTB over a two month period

- to compare data recorded by the data loggers to the self-reported compliance of the subjects using the brushing diaries

- to compare the level of compliance of a group of subjects at two time points, 4 months apart.

\section{MATERIALS AND METHODS}

Compliance with oral healthcare instructions for use of the powered toothbrush was assessed on two occasions. On the first 
occasion data loggers and brushing diaries were used and on the second only loggers recorded compliance. The two periods over which compliance was recorded were four months apart. Ethical approval for the study was granted by the Joint Ethics Committee of Newcastle and North Tyneside, UK.

\section{Subjects}

Subjects who had been referred to the Department of Periodontics at the Newcastle Dental Hospital, UK were recruited. The subjects who all suffered from chronic periodontitis were given a powered toothbrush (PTB) as part of the overall management of their periodontal disease.

\section{Powered toothbrush}

Philips Jordan Sensiflex 2000 ${ }^{\circledR}$ PTBs (HX2550) were modified to carry data loggers. There was no difference in either the action of the brush or the brush head design compared to the standard, marketed model. Access to the data logger interface was made by removing the product label on the base of the brush handle and unscrewing the water-tight sealing cap. Subjects were shown the two speed settings available and the LED timer that flashed after 2 minutes' brushing time had elapsed. Subjects were also asked to leave the brush in the charging stand whilst it was not in use.

\section{Data loggers}

The specially designed data logger consisted of a microprocessor, a re-writable memory and a battery with sufficient power to last for a period of up to 2 months. The microprocessor was programmed to collect and store several operating characteristics of the toothbrush sequentially each time it was used:

- brushing time in normal speed setting and brushing time in lower speed setting, the sum of which was the total time the brush was used in a single brushing session

- maximum, minimum and average motor current which are measures for the load to which the motor is subjected, and hence, for the (brushing) force applied to the brush head. Very low currents indicated that the brush had been turned on without being used. Very high currents, in contrast, indicated that the brush had been used vigorously

- the time the brush was (re)-charged (between two consecutive brushing sessions).

The presence of the data logger was not apparent to the subject and affected neither the handling nor the function of the brush. When the brushes were returned after use, each was opened and the data logger connected to a PC-based data acquisition system to download the brushing data for analysis.

\section{Brushing diaries}

Compliance with oral healthcare instructions was also recorded using a conventional, simple tabular brushing diary provided for the subjects to complete every day. Each subject was asked to record the length of toothbrushing time by placing a tick mark in the corresponding box; 1, 2, 3, 4 or 5 minutes.

\section{Oral hygiene instructions}

Standardised toothbrushing instructions were given to all subjects by the same clinical investigator. The advice was to brush for 2 minutes in the morning and 2 minutes in the evening. A 2 minute brushing regimen was chosen as this fitted with the generally accepted advice by the dental profession and with the timer supplied in the powered toothbrush. Subjects were specifically shown the LED on the brush handle that flashed when 2 minutes had elapsed.

\section{Design}

Baseline

Each subject was allocated a data logger PTB, instructed how to operate the brush and given instructions in the use of the brush and specifically, the exact time for which they should brush. They were asked to leave the brush in the charging stand when it was not in use and not to allow other members of the household to use the brush. The subjects were informed at this stage that the brushes contained 'a monitoring device which recorded brushing performance'. A brushing diary was provided with instructions on how it should be completed prior to collection. Finally, an appointment was arranged for 1 month later.

\section{Month 1}

Toothbrushing instructions were reinforced according to need. The first brushing diary was collected, a new diary was provided for the following month. Subjects were asked to bring the diary and their PTB data logger with them to the next visit.

\section{Month 2}

The brushing diary and the PTB data logger were both collected. A replacement, unmodified PTB without data logger was provided for each subject to use for the following 4 months.

\section{Month 6}

Subjects were asked to bring in their PTBs to this visit. These were collected and the subjects again given PTBs with data loggers to use for a further two months. Each subject's conventional PTB was retained and stored in preparation for month 8. Toothbrushing instructions were reinforced. Subjects were asked to bring this brush back with them at the next visit.

\section{Month 8}

The data loggers were collected.

This design ensured that each subject would have two episodes of two months brushing activity recorded by the data loggers. The single episode of two months' brushing activity recorded using the brushing diaries co-incided with the first period during which the data logger was used and the period during which the periodontal treatment was provided. The second period, for all subjects, coincided with the programme of supportive periodontal care.

Data were retrieved from the data loggers using a PC interface and Labview ${ }^{\circledR}$ software. The data were then imported into an Excel spreadsheet for analysis. Events from the brushing diaries were keyed manually into an Excel spreadsheet to evaluate the reported compliance with toothbrushing instructions.

Data from each data logger were evaluated by dot plotting to determine the pattern of brushing time at high or low speed, charging time and average current per brushing event. Simple descriptive statistics were used to evaluate the brushing events from the data loggers. Brushing events were categorised into one of the following three groups:

- Compliant: Brushing event of between 120-130 s (recommended time $120 \mathrm{~s}$ )

- Partially compliant: Brushing event of 90-119 s or 131-150 s

- Non-compliant: Brushing event of $<90$ s or $>150$ s

Table 1 Percentage distribution of the combined diary events. Standard error and $95 \%$ confidence intervals calculated from the percentage of diary events per subject.

Reported brushing time Percentage of all events SE mean $95 \% \mathrm{Cl}$ mean

1 minute 2 - 2 - 2 to 3

2 minutes

3 minutes

4 minutes

5 minutes

$\begin{array}{rlc}2 & 1 & -0.2 \text { to } 3 \\ 58 & 9 & 35 \text { to } 72 \\ 18 & 5 & 4 \text { to } 23 \\ 7 & 5 & 3 \text { to } 22 \\ 5 & 7 & 5 \text { to } 34\end{array}$


Table 2 Data logger events given as percentages for individual subjects categorized into 5 levels of compliance according to brushing time (seconds). Data for month 0-2 and month 6-8 are shown.

\begin{tabular}{|c|c|c|c|c|c|c|c|c|c|c|}
\hline \multicolumn{6}{|c|}{ Month 0-2 } & \multicolumn{5}{|c|}{ Month 6-8 } \\
\hline Subject & $<90$ & 90-119 & $120-130$ & $131-150$ & $>150$ & $<90$ & 90-119 & $120-130$ & $131-150$ & $>150$ \\
\hline 1 & 3 & 1 & 82 & 11 & 2 & - & - & - & - & - \\
\hline 2 & 7 & 0 & 71 & 17 & 4 & 0 & 5 & 64 & 30 & 5 \\
\hline 3 & 8 & 21 & 64 & 5 & 2 & - & - & - & - & - \\
\hline 4 & 7 & 10 & 56 & 20 & 6 & 1 & 5 & 52 & 35 & 7 \\
\hline 5 & 25 & 6 & 36 & 22 & 12 & - & - & - & - & - \\
\hline 6 & 34 & 0 & 18 & 15 & 34 & ns & ns & ns & ns & ns \\
\hline 7 & 56 & 15 & 12 & 8 & 10 & - & - & - & - & - \\
\hline 8 & 27 & 7 & 9 & 13 & 44 & 26 & 30 & 19 & 9 & 16 \\
\hline 9 & 31 & 24 & 8 & 9 & 28 & - & - & - & - & - \\
\hline 10 & 53 & 10 & 5 & 8 & 24 & 53 & 11 & 22 & 11 & 3 \\
\hline 11 & 82 & 9 & 5 & 0 & 4 & - & - & - & - & - \\
\hline 12 & 3 & 1 & 3 & 7 & 86 & 9 & 7 & 4 & 7 & 73 \\
\hline 13 & 34 & 6 & 0 & 13 & 47 & 7 & 0 & 4 & 0 & 89 \\
\hline 14 & 0 & 0 & 0 & 0 & 100 & 0 & 2 & 2 & 3 & 93 \\
\hline 15 & - & - & - & - & - & 0 & 0 & 96 & 4 & 0 \\
\hline 16 & - & - & - & - & - & 23 & 14 & 29 & 18 & 16 \\
\hline 17 & - & - & - & - & - & ns & ns & ns & ns & ns \\
\hline
\end{tabular}

Events recorded in the brushing diaries were also pooled. As these events were whole minute increments $(1,2,3,4$ or 5 minutes) they were categorised as being either compliant ( 2 minutes) or non-compliant (1, 3, 4 or 5 minutes).

The proportions of compliant and non-compliant events were calculated for both the data logger and brushing diary data. Ninety-five per cent confidence intervals and SE for the mean percentage in each category were calculated using single-sample $t$-tests.

\section{OBSERVATIONS}

Eighteen data logger toothbrushes were allocated. One subject did not return for the appointment at month two and ignored repeated requests to return the data logger toothbrush. Three data loggers failed to record any data. At month 6, fifteen data logger brushes were available to allocate to the subjects and all of these were returned at month 8 . Six loggers failed to record any data. Therefore, data from 14 data loggers for months $0-2$ and nine data loggers for months 6-8 were available.

A total of 2,333 brushing episodes were recorded in the diaries. Fifty-eight per cent of events were for the suggested 2 minutes brushing time, $2 \%$ for 1 minute, 18\% for 3 minutes, $7 \%$ for 4 minutes and 15\% for 5 minutes. The subjects' reported non-compliance using the diaries was $42 \%$ with only $2 \%$ of all events recording less time than the recommended 2 minutes brushing. Table 1 shows the percentage distribution of all events with SE and 95\% confidence intervals for the mean determined by a single sample t-test.

Fourteen data loggers recorded a total of 2,287 brushing events during months $0-2$. Three loggers did not record any information. Table 2 shows the percentage distribution of events in the 5 categories of compliance for each subject (1-17). The data are arranged to show the largest to the smallest proportions recorded of compliance (120-130 s) for the 0-2 month recordings. The percentage distribution of the combined data logger events, SE and 95\% CI for the group means in each category are shown in Table 3. Overall, $34 \%$ of the events recorded compliance with 2 minutes brushing (120-130 s), 20\% of events were partially compliant within 30 seconds of the suggested brushing time; $8 \%$ under and 12\% over. Forty-six per cent of events recorded brushing episodes of more than 30 seconds from the prescribed time; 23\% under and 23\% over. Individual data logger data in Table 3 show that the range of events recording compliance with 2 minutes brushing was 0-82\%. One data logger recorded that one subject (14) showed non-compliance ( $>150$ s) for $100 \%$ of the events recorded.
Nine data loggers recorded a total of 1,526 events during months 6-8. Six data loggers did not record any information. The subject and combined data are also shown in Tables 2 and 3. Twenty-four per cent of the events were compliant with the recommended 2 minutes brushing, 24\% were partially compliant and $52 \%$ were non-compliant. The range of events recorded as compliant was 2-96\%. Seven sets of data logger recordings were available for both time periods ( $0-2$ months and 6-8 months). The proportion of under non-compliant events reduced for 4 subjects, remained the same for 2 subjects and increased for 1 subject. The proportion of over non-compliant events reduced for 4 subjects and increased for 3 subjects. This net reduction in the non-compliant categories led to increased levels of partial-compliance and compliance within this sub-group.

\section{DISCUSSION}

This study was designed to determine more accurately the level of compliance with toothbrushing instructions as part of a larger controlled clinical trial. Historically investigators have used daily brushing diaries and questionnaires to determine this variable, but detailed analyses of these data were not always presented. ${ }^{11-13,17,18}$ In fact, one group commented that this method of recording compliance data was 'less than ideal'. ${ }^{15}$

The calculation of 95\% confidence intervals (95\% CI) for the mean of each compliance category gives an insight into the differences between the data from the data loggers compared with the diaries. The 95\% CI for the mean percentage of brushing events for under-compliance recorded by the diaries was between $0-3 \%$. Conversely, the interval for mean percentage of events recorded by the data loggers was between $12-40 \%$. With regard to events recorded

Table 3 Percentage distribution of the combined data logger events. standard error and 95\% confidence intervals calculated from the percentage of categorized data logger events per subject.

\begin{tabular}{llccc}
\hline Level of compliance & Period & \% of all events & SE & $95 \% \mathrm{Cl}$ \\
\hline Under non-compliance & 0-2 months & 23 & 7 & 12 to 40 \\
& 6-8 months & 19 & 6 & -0.5 to 27 \\
Under partial-compliance & 0-2 months & 8 & 2 & 3 to 12 \\
& 6-8 months & 10 & 3 & 0.1 to 15 \\
Compliance & 0-2 months & 34 & 8 & 9 to 43 \\
Over partial-compliance & 6-8 months & 24 & 11 & 8 to 57 \\
0-2 months & 12 & 2 & 8 to 4 \\
Over non-compliance & 6-8 months & 14 & 4 & 4 to 22 \\
& 0-2 months & 23 & 8 & 11 to 47 \\
& 6-8 months & 33 & 13 & 3 to 64
\end{tabular}


as compliant (data loggers 9-43\%, diaries 35-72\%) and over-compliant (data loggers 11-47\%, diaries 27-63\%), there appears to be overlap of the confidence intervals suggesting a similarity of the distribution of the data from diaries and data loggers. The spread of the 95\% CI for each category, reflects the small sample under observation and the wide variation between subjects and it would be unwise to draw detailed conclusions from this comparison.

The results appear to have split the study cohort into three almost equal categories. One third of subjects spent less time than prescribed, a third spent the recommended time; and a third spent more than the time suggested. This distribution agrees with the opening statements by Boudes ${ }^{6}$ reviewing compliance with drug therapies.

This is the first study to pilot this technology in a dental trial and clearly, the results show considerable discrepancy from the data recorded manually in brushing diaries supporting that such observations must be appraised with considerable caution. However, there were also some problems encountered with the prototype technology. A number of factors may account for the failure of some of the data loggers: power supply, limitations with space in the brush handle; delicacy of the electronics; and the interface to download recorded data. Reliability will improve with the development of more robust data logger technology, and timeand date-stamp recordings will allow comparisons of data reported using more sophisticated diaries.

We acknowledge, however, that the cohort used in this study is very small and need not necessarily relate accurately to the more general population. The cost of the data loggers was prohibitive to undertaking a larger study but nevertheless, the poor compliance recorded in this small group of patients shows that even those with disease find it difficult to comply accurately with professional advice even over a relatively short period of time. There are of course implications for those in the dental team who are regularly involved in providing oral health advice and, in particular tooth brushing instructions. Most dental professionals have considerable experience of patients whose actual oral healthcare practice appears to be at variance with their reported or self-perceived behaviour and our data only serves to confirm that poor compliance with recommended toothbrushing time may well be widespread amongst dental patients. From the medico-legal standpoint, it is crucial, therefore, for professionals to record precisely the information and recommendations that are given to patients and to make them aware of the potential consequences of failing to follow that advice and that a poor response to treatment may well be their responsibility.

Subjects who consent to participate in clinical investigations must have their rights protected and upheld at all times. To withhold information about the methodology by which they are to be observed would be unethical and divisive, this includes the use of data loggers that are in essence covert observers of an individual's actions. Rand and Sevick ${ }^{28}$ concluded that clinicians and researchers should always remain both reflective and vigilant about the ethical implications of adherence monitoring and promotion, everyone has a 'right to privacy and the principle of autonomy'. Therefore, if a subject is to be fully informed of the experimental procedures prior to consent, a degree of bias towards compliance might be introduced. Subjects who are being watched will tend to do as they are told. The subjects in this study were informed of a device that recorded 'brushing performance', but no reference to brushing time was made to them. We believe this information upheld and respected the subject's best interests, autonomy and privacy, and allowed a valid comparison between compliance recorded using both traditional and new technology.

\section{CONCLUSIONS}

1. This electronic data logger was used successfully to gather compliance information on half of the subjects participating over the entirety of the study

2. The use of manually completed brushing diaries did not provide an accurate reflection of subject compliance in this cohort of dental patients

3. Further development of this technology will need to include date stamping of data for a true comparison to sophisticated brushing diaries.

This study was undertaken in collaboration with, and as part of a PhD project sponsored by Philips Research, Eindhoven, The Netherlands.

1. Bond W, Hussar D. Detection methods and strategies for improving medication compliance. Am J Hospital Pharmacy 1991; 48: 1978-1988.

2. Vander Stichele R. Measurement of patient compliance and the interpretation of randomized clinical trials. Eur JClin Pharmaco/ 1991:41:27-35.

3. Kruse W. Patient compliance with drug treatment - new perspectives on an old problem. Clin Investigator 1992; 70: 163-166.

4. Urquhart J. Role of patient compliance in clinical pharmocokinetics. Clin Phamarcokinetics 1994; 27: 202-215.

5. Wilson J. How patient compliance to suggested oral hygiene and maintenance affect periodontal therapy. Dent Clin North Am 1998; 42: 389-403.

6. Boudes P. Drug compliance in therapeutic trials: a review. Controlled clinical trials 1998; 19: 257-268.

7. Kass M A, Gordon M, Meltzer D W. Can ophthalmologists correctly identify patients defaulting from pilocarpine therapy? Am J Ophthalmol 1986; 101: 524-30.

8. Crammer J A, Scheyer R D, Mattson R H. Compliance declines between clinic visits. Arch Intern Med 1990; 150: 1509-10.

9. Feinstein A R. On white-coat effects and the electronic monitoring of compliance. Arch Intern Med 1990; 150: 1377-8.

10. Gordon P M, Keohane S G, Herd R M. White coat effects. Br Med J 1995; 311: 1704

11. Khocht A, Spindel L, Person P. A comparative clinical study of the safety and efficacy of three toothbrushes. J Periodontol 1992; 63: 603-610.

12. Burch $J G$, Lanese $R$, Ngan P. A two-month study of the effects of oral irrigation and automatic toothbrush use in an adult orthodontic population with fixed appliances. Am J Orthodontics Dentofac Orthopedics 1994; 106: 121-126.

13. Moran J M, Addy M, Newcombe R G. A comparative study of stain removal with two electric toothbrushes and a manual brush. J Clin Dent 1995; 6: 188-193.

14. Cronin M, Demblling W, Warren P R, King D W. A 3-month clinical investigation comparing the safety and efficacy of a novel electric toothbrush (Braun Oral-B 3D Plaque Remover) with a manual toothbrush. Am J Dent 1998; 11: S17-S21.

15. Forgas-Brockmann L B, Carter-Hanson C, Killoy W J. The effects of an ultrasonic toothbrush on plaque accumulation and gingival inflammation. J Clin Periodontol 1998; 25: 375-379.

16. Stalnacke K, Soderfeldt B, Sjodin B. Compliance in use of electric toothbrushes. Acto Odontologica Scand 1995; 53: 17-19.

17. Ainamo J, Xie Q, Ainamo A, Kallio P. Assessment of the effect of an oscillating/rotating electric toothbrush on oral health - A 12-month longitudinal study. J Clin Periodontol 1997; 24: 28-33.

18. Isaacs $R \mathrm{~L}$, et al. A crossover clinical investigation of the safety and efficacy of a new oscillating / rotating electric toothbrush and a high frequency electric toothbrush. Am J Dent 1998; 11: 7-12.

19. Sahota $H$, Landini $G$, Walmsley D. A testing system for electric toothbrushes. Am J Dent 1998; 11: 271-275.

20. Wolff L, Kim A, Nunn M, Bakdash B, Hinrichs J. Effectiveness of a sonic toothbrush in maintenance of dental implants. A prospective study. J Clin Periodontol 1998; 25: 821-828.

21. Crammer J A, Mattson R H, Prevey M L. How often is medication taken as prescribed? A novel assessment technique. J Am Med Assoc 1989; 261: 3273-3277.

22. Averbuch $M$, Weintraub $M$, Pollack D J. Compliance assessment in clinical trails: the MEMS device. J Clin Res Pharmacoepidemio/ 1990; 4: 199-204.

23. Kruse W, Weber E. Dynamics of drug regimen compliance - its assessment by microprocessor-based monitoring. Eur J Clin Pharmacol 1990; 38: 561-565. 